\title{
Seismic Reverse Time Migration Using A New Wave-Field Extrapolator and a New Imaging Condition
}

\author{
Farzad MORADPOURI ${ }^{1}$, Ali MORADZADEH ${ }^{1,2}$, Reynam C. PESTANA $^{3}$, \\ and Mehrdad SOLEIMANI MONFARED ${ }^{1}$
}

\author{
${ }^{1}$ Shahrood University of Technology, Faculty of Mining Petroleum and Geophysics, \\ Shahrood, Semnan, Iran; e-mail: f.moradpouri@gmail.com \\ ${ }^{2}$ School of Mining Engineering, College of Engineering, University of Tehran, \\ Tehran, Iran \\ ${ }^{3}$ Center for Research in Geophysics and Geology (CPGG), \\ Federal University of Bahia (UFBA), Salvador, Brazil
}

\begin{abstract}
Prestack reverse time migration (RTM), as a two way wave-field extrapolation method, can image steeply dipping structures without any dip limitation at the expense of potential increase in imaging artifacts. In this paper, an efficient symplectic scheme, called Leapfrog-Rapid Expansion Method (L-REM), is first introduced to extrapolate the wavefield and its derivative in the same time step with high accuracy and free numerical dispersion using a Ricker wavelet of a maximum frequency of $25 \mathrm{~Hz}$. Afterwards, in order to suppress the artifacts as a characteristic of RTM, a new imaging condition based on Poynting vector and a type of weighting function is presented. The capability of the proposed new imaging condition is then tested on synthetic data. The obtained results indicate that the proposed imaging condition is able to suppress the RTM artifacts effectively. They also show the ability of the proposed approach for improving the amplitude and compensate for illumination.
\end{abstract}

Key words: RTM, Leapfrog-Rapid Expansion Method, Poynting vector, Imaging condition, artifacts. 


\section{INTRODUCTION}

Reverse time migration (RTM) was introduced in the late 1970s (Baysal et al. 1983, McMechan 1983, Whitmore 1983). However, it has been widely used in recent years due to the increasing imaging challenges posed by the complex geological structures and the affordable computational resources. This technique propagates the source wave-field forward and the recorded wave-field backward in time using a two-way wave equation. Afterwards, a proper imaging condition is applied to obtain the subsurface image (Claerbout 1971, Fernandez 2010).

RTM directly solves the two-way (full) acoustic wave equation for wave field propagation, and for this reason it has been recognized as a preferred imaging algorithm in many geologically complex basins, including steeply dipping reflectors and complex structures in complicated velocity models, e.g., below salt bodies (Liu et al. 2011, Costa et al. 2009). RTM allows all waves to propagate in all directions. Thus, undesired cross-correlation of non-reflecting points in the subsurface would be present while the recorded wave-fields are extrapolated backwards in time. It exhibits low-frequency artifacts in the migration results when an imaging condition is applied to these reflections (Guitton et al. 2007, Mulder and Plessix 2004, Fletcher et al. 2005, Xie and Wu 2006, Yoon et al. 2004, Du and Qin 2009). These events are most noticeable in shallow layers or strong wave impedance contrast $(\mathrm{Du}$ and Qin 2009).

\subsection{Wave-field extrapolation}

Wave-field extrapolation is implemented by solving the wave equation through various mathematical approaches. Among the explicit methods there are the following: (i) Finite differences (FDs), which makes use of the Taylor expansion (Etgen 1986); the finite difference method (FDM) is a wellknown and popular numerical tool to discretize the wave equation (Wards et al. 2008). The numerical solution by finite differences of the wave equation uses temporal and spatial discretization. Its use has been common in the approximation of the spatial and time derivatives for a wave-field. Originally, the time operator was approximated by a second-order scheme, whereas the spatial derivatives were approximated by a fourth-order scheme. This form of time derivative approximating may introduce numerical error, leading to distortion of the pulse and numerical dispersion, which can be avoided with small time steps (Araujo et al. 2014); (ii) The two-step explicit marching method, which makes use of a polynomial expansion (Soubaras and Zhang 2008, Zhang and Zhang 2009). Soubaras and Zhang (2008) presented a new method for RTM. The strategy is based on the high order polynomial expansion, which allows using the $\Delta t$ Nyquist (Song 2001); (iii) Rapid expansion 
method (REM), which uses the Chebyshev expansion (Kosloff et al. 1989, Pestana and Stoffa 2010). As an alternative to the FD scheme, REM can be used for a more accurate time integration of the wave equation. It uses concepts similar to the work presented by Tal-Ezer et al. (1987), in which Chebyshev polynomials are incorporated in the REM to expand a cosine operator. When REM is combined with the Fourier method for the spatial derivatives, a highly accurate and numerically stable result can be obtained with less computation than the conventional FD approach (in time) to achieve the same level of accuracy (Pestana and Stoffa 2010). Tessmer (2011) also demonstrated that REM is much more efficient than FD schemes (second order in time), especially if large time steps are used. Pestana and Stoffa $(2009,2010)$ explained how to implement REM approach as a timestepping scheme for small and large time steps. Pestana et al. (2012) also showed that the expansion of the cosine function using Chebyshev polynomials has the same form as the Taylor expansion when a specific analytical expression of the Bessel function is considered. Besides that, using the only two terms of Chebyshev expansion, they obtained the same equations used for the second-order FD time approximation; (iv) Symplectic integrators are another class of methods that can also be used to solve the wave equation in RTM. Araujo et al. (2014) presented a symplectic integrator for wave-field extrapolation called Störmer-Verlet-REM which extrapolates the wave-field and its derivative with a good accuracy and stability.

\subsection{Conventional imaging condition}

After wave-field extrapolation from the source and receiver, respectively, an imaging condition is applied to obtain a subsurface image. The conventional imaging condition consists of the extrapolated source and receiver wavefields defined as follows (Liu et al. 2011).

$$
I(\boldsymbol{x})=\int_{t=0}^{t_{\max }} S(\boldsymbol{x}, t) R(\boldsymbol{x}, t) d t
$$

where $S(x, t)$ is the forward extrapolated source wave-field, $R(x, t)$ is the backward propagated receiver wave-field at location $x$ and time $t$, and $t_{\max }$ is the maximum extrapolation time.

The imaging condition in Eq. 1 cannot account properly for multiply reflected waves, including turning and prismatic waves. It is a nonlinear operation that is not able to distinguish between different kinds of waves and spurious cross-correlation at the imaging step and produces artifacts at nonreflecting points (Fleury 2010). In recent years, more attention has been given to improve the imaging condition and reduce the artifacts (Pestana and dos Santos 2013). To reduce the artifacts, several approaches have been pro- 
posed. Youn and Zhou (2001) used a Laplacian operator as a high-pass filter to obtain the image. Mulder and Plessix (2004) used an iterative migration and a high-pass spatial filter to improve migration result. However, the high pass spatial filter might not lead to good results without losing the desired signals, since the frequency bands of the signals and the counterparts of the artifacts are overlapped. Guitton et al. (2007) used a least-squares attenuation method, but this filtering technique cannot completely attenuate the artifacts. Fletcher et al. $(2005 \mathrm{a}, \mathrm{b})$ introduced a directional damping factor into the wave equation to suppress undesired reflection. Yoon and Marfurt (2006) introduced a Poynting-vector imaging condition that was used by several researchers to suppress the artifacts (Costa et al. 2009, Jin et al. 2014, Araujo et al. 2014, Du and Qin 2009, Chen and Huang 2014). Chattopadhyay and McMechan (2008) explicitly compared the most common imaging conditions, excitation time, cross-correlation and receiver/source-wave-field amplitude ratio to clarify which are viable for recovering accurate amplitudes and which are not. Fei et al. (2010) and Liu et al. (2011) used wave-field decomposition in pre-imaging condition. Yoon et al. (2011) and Dickens and Winbow (2011) decomposed the normal zero-lag RTM image into angle/azimuth bins based on the estimated local propagation direction of the source and receiver wave-fields. Several strategies provided information about the wave-field similarity for different space and/or time lags and they can also be used to discriminate the backscattered energy imaging conditions (Rickett and Sava 2002, Sava and Fomel 2006, Sava and Vasconcelos 2011). Whitmore and Crawley (2012) presented a new imaging method based on two images: one is the product of the time derivatives of source and receivers wave-fields and another is the product of the spatial gradients of source and the receivers' wave-fields. These images are then combined to produce a final image. This method is called inverse scattering image that is based on a generalized inverse scattering theory (Stolk et al. 2009). Pestana and dos Santos (2013) used the impedance sensitivity kernel instead of the conventional cross-correlation RTM imaging condition to attenuate low frequency artifacts. Moreover, several researchers tried to improve the amplitudes in RTM based on illumination compensation with different kinds of stabilization (Valenciano and Biondi 2003, Kaelin and Guitton 2006, Vivas and Pestana 2007).

In this study, we firstly propose a new accurate wave-field extrapolator scheme which is called Leapfrog-rapid expansion method (L-REM). This symplectic scheme also calculates the first derivative of the wave-field in the same time step with high accuracy that can be used to calculate Poynting vectors. In the second step, we propose a new imaging condition based on wave-field separation and a weighting function using Poynting vector to suppress the RTM artifacts. Finally, it is shown that the proposed imaging 


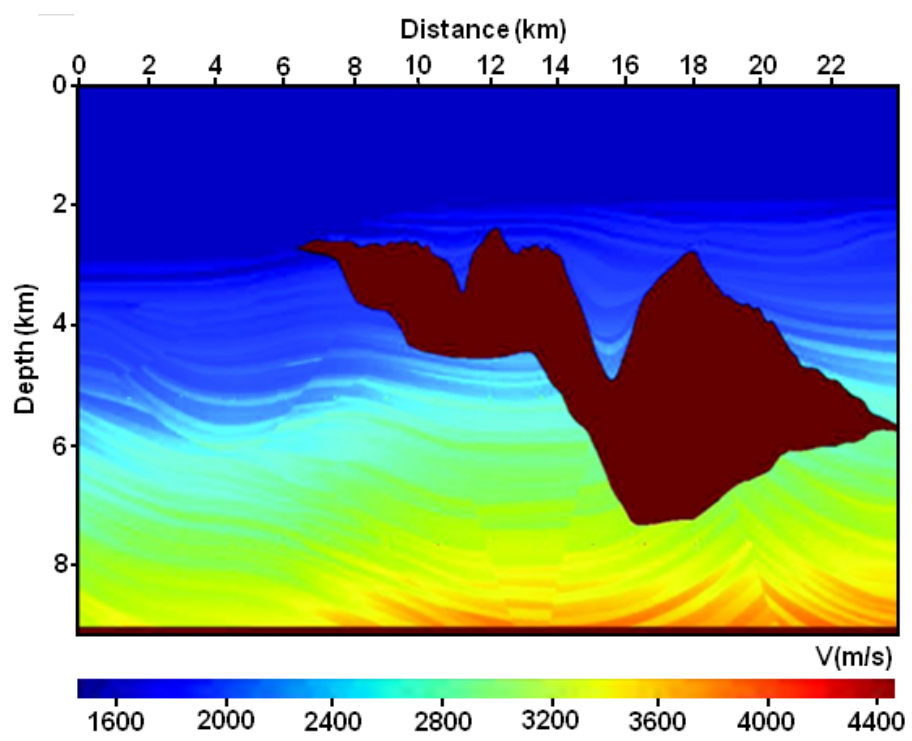

Fig. 1. Velocity variation of the Sigsbee2A model (released by the SMAART JV Consortium 2001).

condition can improve the amplitude and compensate for illumination. These concepts are illustrated by numerical examples using the Sigsbee2A model (Fig. 1). Sigsbee2A model was released by the SMAART JV Consortium as a constant density acoustic velocity model, pre-acoustic velocity model and pre-stack dataset. Its illumination issues are associated with salt structures (Fig. 1). This model includes a complex subsalt media, whose base exhibits flat and steep dip structures. It also exhibits diffraction points, faults and subsalt structures (Fernandez 2010).

\section{SYMPLECTIC SCHEME FOR WAVE-FIELD EXTRAPOLATION}

Most of the migration methods have been derived from the wave equation solution. The difference between the methods is due to the methodology used to solve the wave equation and the type of imaging condition. A threedimensional wave equation for the pressure field is given by the following expression:

$$
\frac{1}{c^{2}} \frac{\partial^{2} P}{\partial t^{2}}=\frac{\partial^{2} P}{\partial x^{2}}+\frac{\partial^{2} P}{\partial y^{2}}+\frac{\partial^{2} P}{\partial z^{2}}
$$

Rewriting Eq. 2 we have:

$$
\frac{\partial^{2} P}{\partial t^{2}}=c^{2} \nabla^{2} P=-L^{2} P
$$


where $P(x, y, z, t)$ denotes the pressure wave-field, $\nabla^{2}$ is the Laplacian operator in Cartesian coordinates, $c(x, y, z)$ is the velocity of propagation and also $c^{2} \nabla^{2}=L^{2}$.

By the way, a Hamiltonian system is a system of equations in the following form (Arnold 1989):

$$
\frac{d \boldsymbol{x}}{d t}=\frac{\partial H}{\partial \boldsymbol{p}}=\boldsymbol{p}, \quad \frac{d \boldsymbol{p}}{d t}=-\frac{\partial H}{\partial \boldsymbol{x}}=\boldsymbol{f}
$$

where $\boldsymbol{x}, \boldsymbol{p}$, and $\boldsymbol{f}$ are $n$-dimensional vectors of the generalized coordinates, momentum and force respectively; $t$ is the independent time variable and $H=H(\boldsymbol{x}, \boldsymbol{p})$ is the Hamiltonian function. The set of position and momentum coordinates $(\boldsymbol{x}, \boldsymbol{p})$ is called the canonical coordinates.

We can also rewrite Eq. 3 using the Hamiltonian formulation as (Bonomi et al. 1998):

$$
\frac{\partial P}{\partial t}=Q, \quad \frac{\partial Q}{\partial t}=c^{2} \nabla^{2} P .
$$

The solution of Eq. 5 using the following initial conditions is given by Eq. 7:

$$
\begin{gathered}
P(x, z, t=0)=P_{0} \quad \text { and } \quad\left[\frac{\partial P(x, z, t)}{\partial t}\right]_{t=0}=\dot{P}_{0}, \\
P(x, z, t)=\cos (L t) P_{0}+\frac{\sin (L t)}{L} \dot{P}_{0} .
\end{gathered}
$$

The wavefields $P(x, z, t+\Delta t)$ and $P(x, z, t-\Delta t)$ can be developed using Eq. 7. Adding these two wave-fields results in:

$$
P(t+\Delta t)+P(t-\Delta t)=2 \cos (L \Delta t) P(t) .
$$

RTM has also been implemented through the analytical solution of wave equation and rapid expansion method presented by Kosloff et al. (1989), based on Tal-Ezer et al. (1987) expansion method. Using REM, an efficient orthogonal polynomial series expansion for the cosine function in Eq. 8 is given as follow (Tal-Ezer et al. 1987, Pestana and Stoffa 2010).

$$
\cos (L \Delta t)=\sum_{k=0}^{M \rightarrow \infty} C_{2 k} J_{2 k}(\Delta t R) Q_{2 k}(f) \quad \text { where } f=\frac{i L}{R}
$$

where $C_{2 k}=1$ for $k=0$ and $C_{2 k}=2$ for $k \neq 0, J_{2 k}$ is the Bessel function of order $2 k$ and $Q_{2 k}$ are the modified Chebyshev polynomials.

The value of $R$ is given by: 


$$
R=\pi c_{\max } \sqrt{1 / \Delta x^{2}+1 / \Delta z^{2}},
$$

where $R$ is a scalar larger than the range of $L^{2}$ eigenvalues in which $c_{\max }$ is the maximum velocity in the grid, $\Delta x$ and $\Delta z$ are the grid spacing (Tal-Ezer et al. 1987).

Expression 9, contains only even modified Chebyshev polynomials that satisfy the following recurrence relations.

$$
Q_{2 k+2}(f)=2\left(1-2 f^{2}\right) Q_{2 k}(f)-Q_{2 k-2}(f) .
$$

Thus, the recursion starts with:

$$
Q_{0}(f)=1, Q_{2}(f)=1-2 f^{2} .
$$

The summation of Eq. 9 converges exponentially for $M>\Delta t R$; therefore, it can be safely truncated using a value of $M$ slightly greater than $\Delta t R$ (Tal-Ezer et al. 1987). Pestana and Stoffa (2010) demonstrated that when $M=1$, only two terms are kept in the summation. This approximation of the cosine function using the Chebyshev polynomials provides a 2 nd-order finite difference scheme. When $M=2$, the $L^{4}$ operator term is included and the approximation is equivalent to the 4th-order finite-difference scheme proposed by Dablain (1986) and Etgen (1986).

Using REM in Eq. 8, we have:

$$
P(t+\Delta t)+P(t-\Delta t)=2\left[\sum_{k=0}^{M \rightarrow \infty} C_{2 k} J_{2 k}(\Delta t R) Q_{2 k}\left(\frac{i L}{R}\right)\right] P(t) .
$$

On the other hand, a symplectic integrator (SI) is a numerical integration scheme for a specific group of differential equations relating to classical mechanics and symplectic geometry (Yoshida 1990). Symplectic integrators form a subclass of geometric integrators that are canonical transformations (transformations that preserve the Hamilton equations of motion (Deriglazov and Filgueiras 2009)). The wave Eq. 5 allows us to use a family of symplectic methods for the integration in time. According to Skell et al. (1997), symplectic methods preserve a certain invariant of the Hamiltonian system and are stable for systems in which the linear extrapolation step size is sufficiently small.

Bonomi et al. (1998) performed a time integration (Eq. 5) using a third order symplectic scheme presented by Sexton and Weingarten (1992). The numerical solution to the wave equation of Bonomi et al. (1998), called leapfrog, that is given by the following expressions: 


$$
\begin{aligned}
Q^{\left(n+\frac{1}{3}\right)} & =Q^{(n)}+\frac{1}{6} \Delta t c^{2} \nabla^{2} P^{(n)}, \\
P^{\left(n+\frac{1}{2}\right)} & =P^{(n)}+\frac{1}{2} \Delta t Q^{\left(n+\frac{1}{3}\right)}, \\
Q^{\left(n+\frac{2}{3}\right)} & =Q^{\left(n+\frac{1}{3}\right)}+\frac{2}{3} \Delta t c^{2} \nabla^{2} P^{\left(n+\frac{1}{2}\right)}, \\
P^{(n+1)} & =P^{\left(n+\frac{1}{2}\right)}+\frac{1}{2} \Delta t Q^{\left(n+\frac{2}{3}\right)}, \\
Q^{(n+1)} & =Q^{\left(n+\frac{2}{3}\right)}+\frac{1}{6} \Delta t c^{2} \nabla^{2} P^{(n+1)},
\end{aligned}
$$

According to Bonomi et al. (1998), Eq. 14 allows an improvement in the accuracy, without increasing the memory requirement in relation to the method of central finite differences of the second order and preserves the time reversibility of the wave equation. This scheme also provides directly the time derivative of the wave-field $(Q)$, which will be useful for calculating the Poynting vector.

For scheme 14, the maximum time sampling to march the wave-field in a stable way must satisfy the following condition:

$$
\Delta t \leq \sqrt{6} /\left(c_{\max } \pi \sqrt{\left(\frac{1}{\Delta x}\right)^{2}+\left(\frac{1}{\Delta z}\right)^{2}}\right) .
$$

According to Araujo et al. (2014), by adding the term $-2 P(t)$ on both sides of Eq. 13 and multiplying by $1 / \Delta^{2}$, a second order central finite differences operator is calculated as follows:

$$
\frac{P^{(n+1)}-2 P^{(n)}+P^{(n-1)}}{(\Delta t)^{2}}=\frac{\partial^{2} P}{\partial t^{2}}=W\left(P^{(n)}\right),
$$

On the basis of Eqs. 13 and 16, we can rewrite the $W\left(P^{(n)}\right)$ as follows:

$$
W\left(P^{(n)}\right)=\frac{2}{(\Delta t)^{2}}\left[\sum_{k=0}^{M} C_{2 k} J_{2 k}(\Delta t R) Q_{2 k}\left(\frac{i L}{R}\right)-1\right] P(t) .
$$

Using Eq. 16 and the Hamiltonian formulation, we have:

$$
\frac{\partial P}{\partial t}=Q, \quad \frac{\partial Q}{\partial t}=W\left(P^{(n)}\right)
$$

In this paper, we propose a solution for Eq. 18 using the leapfrog method and REM as follows: 


$$
\begin{aligned}
& P^{(n+1)}=P^{(n)}+(\Delta t) Q^{(n)}+ \frac{1}{3}\left[\sum_{k=0}^{M} C_{2 k} J_{2 k}(\Delta t R) T_{2 k}\left(\frac{i L}{R}\right)-1\right] P^{(n)}, \\
& Q^{(n+1)}=Q^{(n)}+\frac{1}{(\Delta t)}\left(\sum_{k=0}^{M} C_{2 k} J_{2 k}(\Delta t R) T_{2 k}\left(\frac{i L}{R}\right)-1\right) \times \\
& \times\left(2 P^{(n)}+\frac{1}{6}\left(P^{(n+1)}-2 P^{(n)}+P^{(n-1)}\right)\right) .
\end{aligned}
$$

The new numerical scheme 19 is called Leapfrog-REM. This scheme provides both the wave-field $\left(P^{(n+1)}\right)$ and its derivative $\left(Q^{(n+1)}\right)$ with respect to time. Furthermore, it is interesting to note that the calculated wavefield $\left(P^{(n+1)}\right)$ is used in the same iteration to calculate $Q^{(n+1)}$. Using this information, we are able to calculate the Poynting vector. Moreover, the Poynting vector information can also be used to separate the wave-field in its up-going and down-going components and to calculate the reflection angles. This information is used to improve the imaging condition which will be addressed in the later sections.

\subsection{Numerical analysis}

In order to verify the numerical accuracy and behaviour of the error associated with L-REM scheme (Eq. 19), a numerical example is presented to be solved using different time sampling $(\Delta t)$ values for wave-field extrapolation. To implement this, an explosive source is used in the centre of the computational domain having a Ricker wavelet with a maximum frequency of $25 \mathrm{~Hz}$.

According to Chen (2009) for small value of $\Delta t$, the numerical solution converges to the analytical solution. Based on this idea, we used the solution of L-REM scheme 19 using $\Delta t=1 \mathrm{~m} \mathrm{~s}$ as a reference solution for comparison. Then, the normalized amplitude of the wave-fields in a specific point of the computational domain was compared with the reference solution. In Fig. 2, the normalized amplitude curves were computed for different time sampling using the L-REM scheme. As can be seen, the numerical results are almost the same and indistinguishable for increasing time sampling compared with the results of $\Delta t=1 \mathrm{~m} \mathrm{~s}$ at the same position.

Absolute error associated with time sampling increase is shown in Fig. 3a-c. The results prove a high accuracy of extrapolation for $\Delta t=2 \mathrm{~m} \mathrm{~s}$ (Fig. 3a). Also, it is interesting to note that when $\Delta t$ is increased to $\Delta t=$ $4 \mathrm{~ms}$ and $\Delta t=8 \mathrm{~m} \mathrm{~s}$ (Fig. $3 \mathrm{~b}$ and c), the proposed scheme still provides a good level of accuracy compared with the results using $\Delta t=1 \mathrm{~m} \mathrm{~s}$ and $\Delta t=2 \mathrm{~m} \mathrm{~s}$. This is the main problem in many schemes, like finite difference methods. In such methods the time step increase leads to numerical dispersion. The obtained results indicate that maximum value of the amplitude er- 


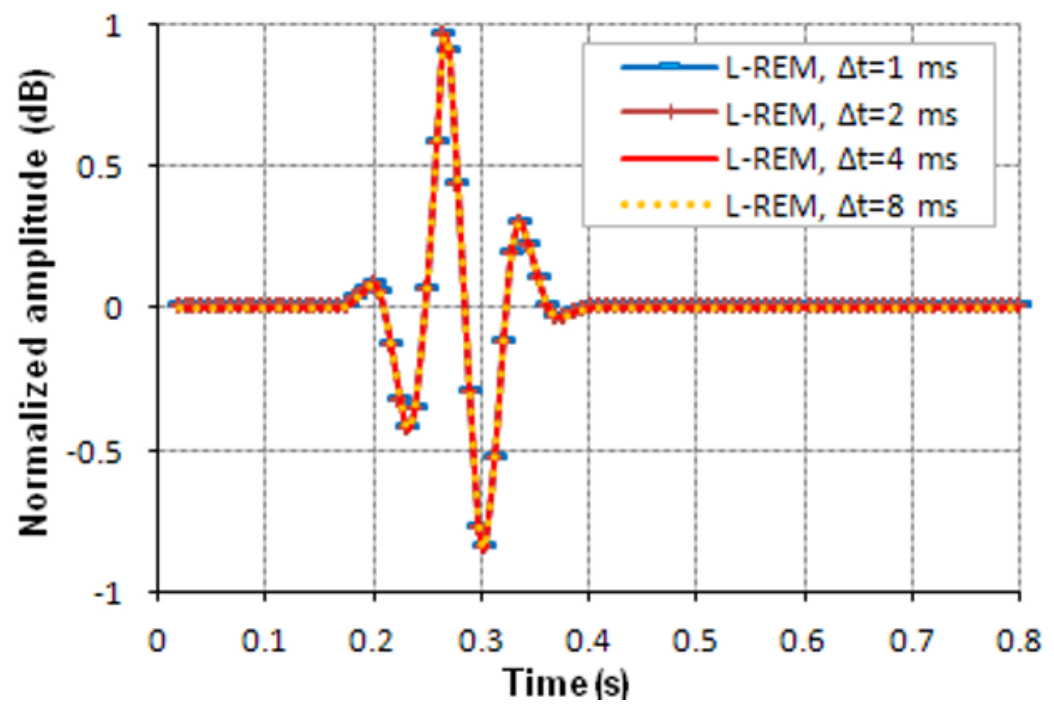

Fig. 2. The normalized amplitude of seismic traces for different time sampling using L-REM scheme (Eq. 19). The normalized amplitude for $\Delta t=1 \mathrm{~m} \mathrm{~s}$ is considered as a reference solution.
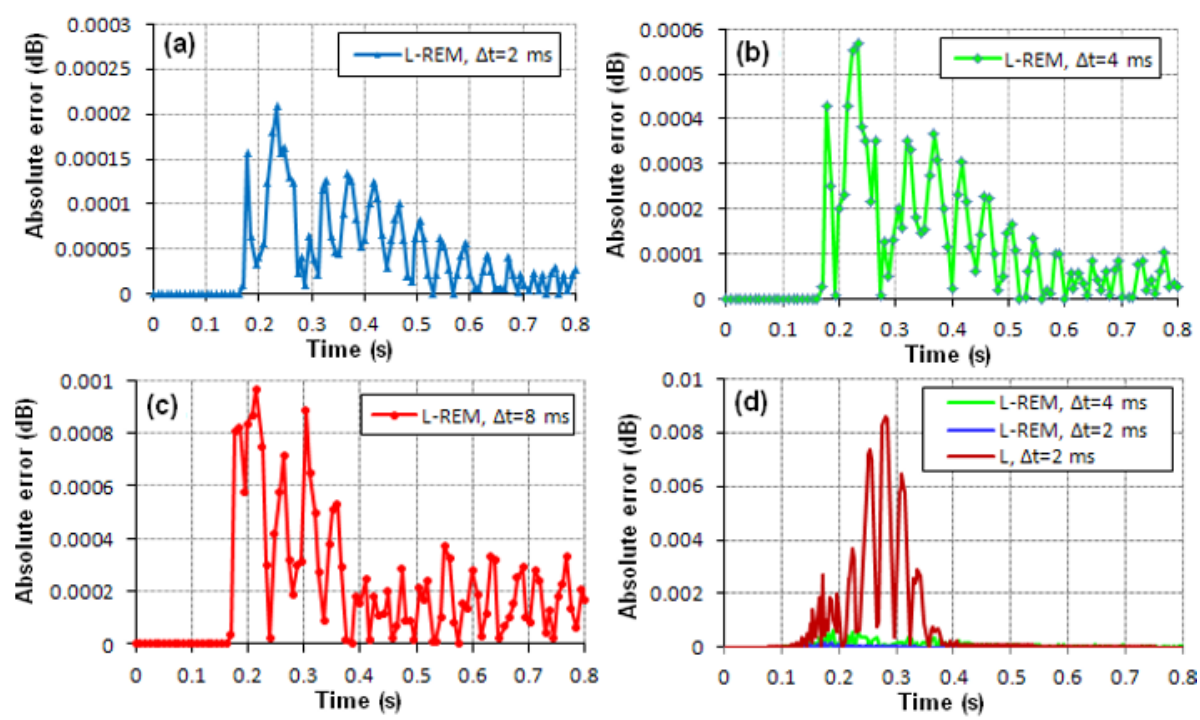

Fig. 3. Absolute error of the normalized amplitude for seismic traces shown in Fig. 2, for time sampling: (a) $\Delta t=2 \mathrm{~m} \mathrm{~s}$, (b) $\Delta t=4 \mathrm{~m} \mathrm{~s}$, and (c) $\Delta t=8 \mathrm{~m} \mathrm{~s}$. The L-REM normalized trace for $\Delta t=1 \mathrm{~m} \mathrm{~s}$ was considered as reference; (d) comparison of the L-REM and Leapfrog (L) error for different time steps. 
ror occurs when $\Delta t=8 \mathrm{~m} \mathrm{~s}$ using L-REM scheme, while this value still shows a high accuracy of computation. It is notable that the computational cost of L-REM procedure is negligible compared to the similar schemes (Tessmer 2011). Although small time steps are needed to avoid numerical dispersion, it increases computation cost. On the other hand, larger time steps reduce the accuracy. Using the proposed scheme in Eq. 19, we can extrapolate the wave-field for larger time steps accurately with reasonable computation cost and free of dispersion. Figure $3 \mathrm{~d}$ shows the error of L-REM scheme and a rather similar method called Leapfrog (L) in Eq. 14 which is much more accurate than finite difference (FD) methods. As can be seen, even for larger time step of $\Delta t=4 \mathrm{~m} \mathrm{~s}$ the proposed scheme 19 shows a high accuracy versus Leapfrog scheme.

\section{NEW IMAGING CONDITION}

In pre-stack RTM algorithm, the conventional imaging condition (Eq. 1) correlates all kinds of waves including direct, prismatic and turning waves which are from non-reflecting points. Correlation of these waves causes a set of artifacts to appear in the migrated image (Fig. 4).

In this study, a new imaging condition is introduced that includes both wave-field separation and a weighting function based on Poynting vectors. The Poynting vectors describe the energy flux density and can be used to separate the wave-fields and to calculate the reflection angles. Yoon et al. (2004) calculated Poynting vectors as a product of the time derivative and the gradient of the wave-field as follows:

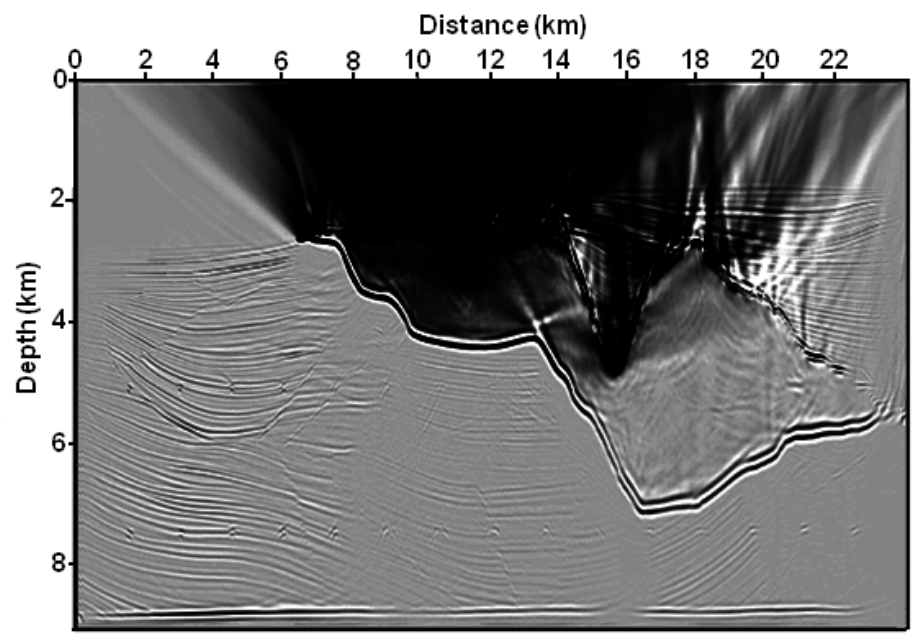

Fig. 4. RTM result using L-REM scheme 19 and zero-lag cross-correlation imaging condition (Eq. 1). 


$$
\mathbf{J}=-\frac{\partial P}{\partial t} \nabla P,
$$

where $\mathbf{J}$ is the Poynting vector and $P$ is the wave field pressure at a spatial location $x$ and time $t$.

As mentioned previously, the L-REM scheme has been introduced for solution of wave equation and its derivative in time. We also used a central FD scheme to compute the gradient vector (first-order spatial derivatives). Then Poynting vectors were calculated using Eq. 20.

Calculating of the Poynting vectors allows us to separate the wave-fields and to calculate the reflection angles. These are used in the new presented imaging condition to suppress the artifacts which given as follows:

$$
I(\boldsymbol{x})=\frac{\int_{0}^{t_{\max }}\left[S_{d}(\boldsymbol{x}, t) R_{u}(\boldsymbol{x}, t)+S_{u}(\boldsymbol{x}, t) R_{d}(\boldsymbol{x}, t)\right] W(\theta) d t}{\int_{0}^{t_{\max }} S^{2}(\boldsymbol{x}, t)},
$$

where $S_{d}(\boldsymbol{x}, t), S_{u}(\boldsymbol{x}, t)$, and $R_{d}(\boldsymbol{x}, t), R_{u}(\boldsymbol{x}, t)$ are the down-going and up-going separated wave-field components for source and receiver, respectively. $S^{2}(\boldsymbol{x}, t)$ is the source normalizing term, $W(\theta)$ denotes weighting function and $\theta$ is the reflection angle that is defined as the half angle between the incident wave and the reflected wave $(\gamma)$. It can be obtained using the following equation:

$$
\gamma=2 \theta=\arccos \frac{\mathbf{J}_{\mathrm{S}} \cdot \mathbf{J}_{\mathrm{R}}}{\left|\mathbf{J}_{\mathrm{S}}\right|\left|\mathbf{J}_{\mathrm{R}}\right|},
$$

where $\mathbf{J}$ is the Poynting vector.

Araujo et al. (2014) presented an approach to separate the wave-field to down-going and up-going components. They calculated the direction of propagation of the wave-fields through the Poynting vector using Eq. 23:

$$
\beta=\arctan \left(\frac{\mathbf{J}_{\mathbf{Z}}}{\mathbf{J}_{\mathbf{x}}}\right),
$$

where $\beta$ is the angle of direction for wave-field propagation. $\mathbf{J}_{\mathbf{x}}$ and $\mathbf{J}_{\mathrm{z}}$ denote horizontal and vertical Poynting vectors, respectively. The up-going part of the wave-field is considered when $\beta \in[0, \pi]$, and the down-going part of the wave-field is considered when $\beta \in[0,-\pi]$.

Equation 23 allows us to separate the wave-field to the down-going and up-going components. As can be seen in Fig. 5a, cross-correlation of the separated wave-fields attenuates the artifacts. However, still cross-correlated backscatters from the non-reflection points can produce the artifacts which 

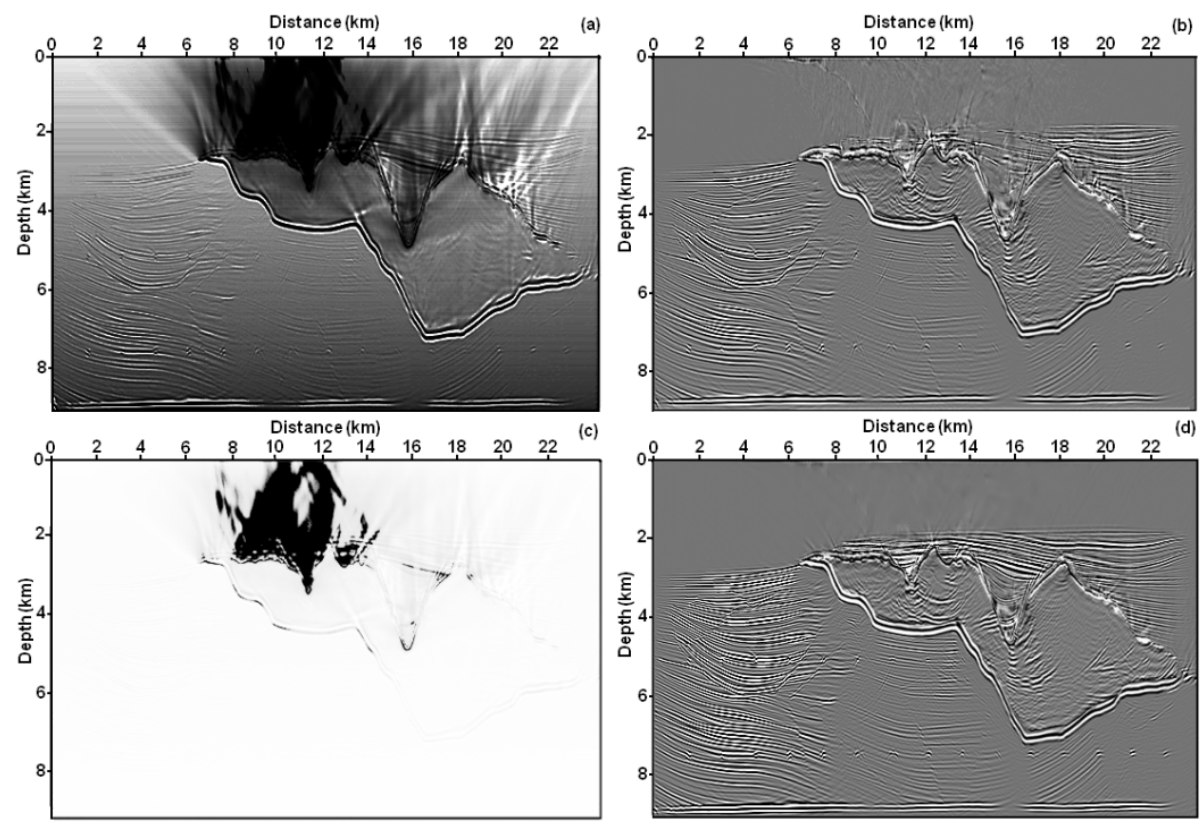

Fig. 5. RTM results using L-REM scheme 19 and applying the new imaging condition (Eq. 21): (a) without the weighting function $W(\theta)$ for $0 \leq \theta \leq 90^{\circ}$, (b) without the weighting function $W(\theta)$ for $0 \leq \theta \leq 60^{\circ}$, (c) without the weighting function $W(\theta)$ for angle range $61 \leq \theta \leq 90^{\circ}$, and (d) with the weighting function $W(\theta)$ for $0 \leq \theta \leq 90^{\circ}$.

start to appear for the reflection angle $>60^{\circ}$. Figure $5 \mathrm{~b}$ and $\mathrm{c}$ show the RTM results after wave-field separation using the symplectic scheme 19. It was implemented using the new imaging condition (Eq. 21) without the weighting function for the reflection angles from $0^{\circ}$ to $60^{\circ}$ and from $61^{\circ}$ to $90^{\circ}$, respectively. As can be seen in Fig. $5 \mathrm{~b}$, for angle range $\leq 60^{\circ}$, the artifacts were suppressed fairly well, but some desired information above the upper part of the model is lost. On the other hand, using angle range of $61^{\circ}$ to $90^{\circ}$ causes large amount of artifacts including cross-correlation of non-reflecting points in Fig. 5c.

Considering this, we used a weighting function $W(\theta)$ to have the most likely desired information and to suppress the artifacts for the angle range from $61^{\circ}$ to $90^{\circ}$. This weighting function is described as follows:

$$
W(\theta)=\left\{\begin{array}{ll}
1 & \text { if } 0 \leq \theta \leq 60^{\circ} \\
\cos ^{n}(\theta) & \text { if } 60^{\circ}<\theta \leq 90^{\circ} \forall n=1,3 / 2,2
\end{array} .\right.
$$


This weighting function, besides suppressing the artifacts, has the capability to preserve the cross-correlation of reflecting points in angle range of $61^{\circ}$ to $90^{\circ}$. This is achieved by dividing the angle range to a triplet domain from $61^{\circ}$ to $70^{\circ}, 71^{\circ}$ to $80^{\circ}$ and $81^{\circ}$ to $90^{\circ}$ where each part has the weight of $\cos \theta, \cos ^{3 / 2} \theta$, and $\cos ^{2} \theta$, respectively. Figure $5 \mathrm{~d}$ shows the final migrated image using the symplectic scheme 19 and the complete imaging condition in Eq. 21. It should be mentioned that the presented new imaging condition can improve the imaging amplitude and compensate for illumination. Illumination compensation is achieved by normalizing the cross-correlation imaging condition by the source energy at the imaging points.

\section{CONCLUSION}

Wave-field extrapolation for reverse time migration (RTM) implemented by solving the wave equation through various mathematical methods. In this paper, a symplectic scheme based on Leapfrog integrator and rapid expansion method (L-REM) has been firstly proposed to extrapolate the wavefield and its first time derivatives for the same time step. The proposed L-REM scheme was then tested using a Ricker wavelet with a maximum frequency of $25 \mathrm{~Hz}$ which showed a highly accurate and stable scheme. In addition, the first time derivatives accompanied with spatial derivatives were used to calculate the Poynting vectors for later purposes. Secondly, a new imaging condition based on the Poynting vector and a weighting function was proposed to suppress the low frequency artifacts in RTM. We used the Poynting vector as a basis for both wave-field separation to the down- and up-going components and to calculate the reflection angles which was used to build an efficient weighting function. The obtained results indicate that the introduced weighting function, besides suppressing the artifacts created in the range of $61^{\circ}$ to $90^{\circ}$, has enough capability to preserve the crosscorrelation from reflecting points for the aforementioned angle range. This is performed by dividing angle range to a triplet domain of $61^{\circ}$ to $70^{\circ}, 71^{\circ}$ to $80^{\circ}$ and $81^{\circ}$ to $90^{\circ}$ that each part has the weight of $\cos \theta, \cos ^{3 / 2} \theta$, and $\cos ^{2} \theta$, respectively. The new imaging condition for RTM was tested using the synthetic Sigsbee2A model. The acquired results showed the efficiency of the new imaging condition to suppress the low frequency artifacts, improve the imaging amplitude and compensate for illumination. 


\section{References}

Araujo, E.S., R.P. Pestana, and A.W.G. Santos (2014), Symplectic scheme and the Poynting vector in reverse-time migration, Geophysics 79, 5, 1-10, DOI: 10.1190/geo2013-0303.1.

Arnold, V.I. (1989), Mathematical Methods of Classical Mechanics, 2nd ed., Springer.

Baysal, E., D.D. Kosloff, and J.W.C. Sherwood (1983), Reverse-time migration, Geophysics 48, 1, 1514-1524, DOI: 10.1190/1.1441434.

Bonomi, E., L. Brieger, C. Nardone, and E. Pieroni (1998), 3D spectral reverse time migration with no-wraparound absorbing conditions. In: 78th Ann Int. Mtg. SEG, Expanded Abstracts, 1925-1928.

Chattopadhyay, S., and G.A. McMechan (2008), Imaging conditions for prestack reverse-time migration, Geophysics 73, 3, S81-S89, DOI: 10.1190/ 1.2903822 .

Chen, J. (2009), Lax-Wendroff and Nyström methods for seismic modelling, Geophys. Prospect. 57, 6, 931-941, DOI: 10.1111/j.1365-2478.2009.00802.x.

Chen, T., and L. Huang (2014), Imaging steeply-dipping fault zones using elastic reverse-time migration with a combined wave-field separation and Poynting vector imaging condition. In: Proc. 39th Workshop on Geothermal Reservoir Engineering, 24-26 February 2014, Stanford University, Stanford, USA, SGP-TR-202.

Claerbout, J.F. (1971), Toward a unified theory of reflector mapping, Geophysics 36, 3, 467-481, DOI: 10.1190/1.1440185.

Costa, J.C., F.A. Silva, M. R. Alcantara, J. Schleicher, and A. Novais (2009), Obliquity-correction imaging condition for reverse time migration, Geophysics 74, 3, 57-66, DOI: 10.1190/1.3110589.

Dablain, M.A. (1986), The application of high-order differencing to the scalar wave equation, Geophysics 51, 1, 54-66, DOI: 10.1190/1.1442040.

Deriglazov, A.A., and J.G. Filgueiras (2009), Hamiltonian Formulation and Canonical Transformations in Classical Mechanics, Library of Physics Publication, São Paulo.

Dickens, A., and G.A. Winbow (2011), RTM angle gathers using Poynting vectors. In: 81st Ann Int. Mtg., Soc. Expl. Geophys., Expanded Abstracts, 31093113.

Du, Q.Z., and T. Qin (2009), Multicomponent prestack reverse-time migration of elastic waves in transverse isotropic medium, Chin. J. Geophys. 52, 2, 471478, DOI: $10.1002 /$ cjg2.1367.

Etgen, J. (1986), High-order finite-difference reverse time migration with the 2-way non-reflecting wave equation, Stanford Exploration Project 48, 133-146. 
Fei, T.W., Y. Luo, and G.T. Schuster (2010), De-blending reverse-time migration. In: Soc. Expl. Geophys., Technical Program Expanded Abstracts, 29, 31303134.

Fernandez, A.B. (2010), Subsalt seismic imaging illumination study, M.Sc. Thesis, University of Houston.

Fletcher, R.F., P. Fowler, P. Kitchenside, and U. Albertin (2005a), Suppressing artifacts in prestack reverse-time migration. In: 75th Ann Int. Mtg. Soc. Expl. Geophys., Expanded Abstracts, 2049-2051.

Fletcher, R.F., P. Fowler, P. Kitchenside, and U. Albertin (2005b), Suppressing unwanted internal reflections in prestack reverse time migration, Geophysics 71, 6, E79-E82, DOI: 10.1190/1.2356319.

Fleury, C. (2010), Investigating an imaging condition for nonlinear imaging- principles and application to reverse-timemigration artifacts removal. In: Soc. Expl. Geophys., Ann Mtg., Denver 2010, 3338-3343.

Guitton, A., B. Kaelin, and B. Biondi (2007), Least-squares attenuation of reversetime-migration artifacts, Geophysics 72, 1, S19-S23, DOI: 10.1190/ 1.2399367.

Jin, H., G.A. McMechan, and H. Guan (2014), Comparison of methods for extracting ADCIGs from RTM, Geophysics 79, 3, 89-103, DOI: 10.1190/ geo2013-0336.1.

Kaelin, B., and A. Guitton (2006), Imaging condition for reverse time migration. In: 76th Int. Ann Mtg. Soc. Expl. Geophys., Expanded Abstracts, 2594-2597.

Kosloff, D., A.Q. Filho, E. Tessmer, and A. Behle (1989), Numerical solution of the acoustic and elastic wave equations by a new rapid expansion method, Geophys. Prospect. 37, 4, 383-394, DOI: 10.1111/j.1365-2478.1989. tb02212.x.

Liu, F., G. Zhang, S.A. Morton, and J.P. Leveille (2011), An effective imaging condition for reverse-time migration using wavefield decomposition, Geophysics 76, 1, S29-S39, DOI: 10.1190/1.3533914.

McMechan, G.A. (1983), Migration by extrapolation of time-dependent boundary values, Geophys. Prospect. 31, 3, 413-420, DOI: 10.1111/j.13652478.1983.tb01060.x.

Mulder, W.A., and R.E. Plessix (2004), A comparison between one-way and twoway wave-equation migration, Geophysics 69, 6, 1491-1504, DOI: 10.1190/1.1836822.

Pestana, R.C., and A.W.G. dos Santos (2013), RTM imaging condition using impedance sensitivity kernel combined with Poynting vector. In: 13th Int. Congress of the Brazilian Geophysical Society (SBGF), 26-29 August 2013, Rio de Janeiro, Brazil.

Pestana, R.C., and P.L. Stoffa (2009), Rapid expansion method (REM) for time-stepping in reverse time migration (RTM). In: SEG Technical Program Expanded Abstracts 2009, 2819-2823, DOI: 10.1190/1.3255434. 
Pestana, R.C., and P.L. Stoffa (2010), Time evolution of the wave equation using rapid expansion method, Geophysics 75, 4, T121-T131, DOI: 10.1190/ 1.3449091.

Pestana, R.C., P.L. Stoffa, and A.W.G. dos Santos (2012), The relation between finite differences in time and the Chebyshev polynomial recursion. In: $82 n d$ Ann Int. Mtg. Soc. Expl. Geophys., Expanded Abstracts, DOI: 10.1190/ segam2012-0833.1.

Rickett, J.E., and P.C. Sava (2002), Offset and angle-domain common image point gathers for shot-profile migration, Geophysics 67, 3, 883-889, DOI: 10.1190/1.1484531.

Sava, P., and S. Fomel (2006), Time-shift imaging condition in seismic migration, Geophysics 71, 6, S209-S217, DOI: 10.1190/1.2338824.

Sava, P., and I. Vasconcelos (2011), Extended imaging conditions for wave-equation migration, Geophys. Prospect. 59, 1, 35-55, DOI: 10.1111/j.1365-2478. 2010.00888.x.

Sexton, J.C., and D.H. Weingarten (1992), Hamiltonian evolution for the hybrid Monte Carlo algorithm, Nucl. Phys. B 380, 3, 665, DOI: 10.1016/05503213(92)90263-B.

Skell, R.H., G. Zhang, and T. Schlick (1997), A family of symplectic integrators: Stability, accuracy, and molecular dynamics applications, SIAM J. Num. Anal. 18, 203-222, DOI: 10.1137/ S1064827595282350.

Song, J. (2001), The optimized expression of a high dimension function/manifold in a lower dimensional space, Chin. Sci. Bull. 46, 977-984.

Soubaras, R., and Y. Zhang (2008), Two-step explicit marching method for reverse time migration. In: 70th Ann Int. Conference and Exhibition, EAGE, Extended Abstracts.

Stolk, C.C., M.V. de Hoop, and Op't Root (2009), Linearized inverse scattering based on seismic reverse-time migration. In: Proc. the Project Review, Geo-Mathematical Imaging Group - Purdue University.

Tal-Ezer, H., D. Kosloff, and Z. Koren (1987), An accurate scheme for forward seismic modelling, Geophys. Prospect. 35, 5, 479-490, DOI: 10.1111/ j.1365-2478.1987.tb00830.x.

Tessmer, E. (2011), Using the rapid expansion method for accurate time-stepping in modeling and reverse-time migration, Geophysics 76, 4, S177-S185, DOI: 10.1190/1.3587217.

Valenciano, A., and B. Biondi (2003), 2-D deconvolution imaging condition for shot-profile migration. In: 73rd Int. Ann Mtg. Soc. Expl. Geophys., ExpandedAbstracts, 2431-2433.

Vivas, F., and R.P. Pestana (2007), Imaging condition to true amplitude shot-profile migration. In: 77rd Ann. Int. Mtg. Soc. Expl. Geophys., Expanded Abstracts, 2398-2402. 
Wards, B.D., G.F. Margrave, and M.P. Crewes e Lamoureux (2008), Phase-shift time-stepping for reverse-time migration. In: 78th Ann Int. Mtg. Soc. Expl. Geophys., Expanded Abstracts, Las Vegas/EUA.

Whitmore, D.N. (1983), Iterative depth imaging by back time propagation. In: 53rd Ann Int. Mtg. Soc. Expl. Geophys., Expanded Abstracts, 382-385.

Whitmore, N.D., and S. Crawley (2012), Application of RTM inverse scattering imaging conditions. In: 82nd Ann Int. Mtg. Soc. Expl. Geophys., Expanded Abstracts.

Xie, X.B., and R. Wu (2006), A depth migration method based on the full-wave reverse-time calculation and local one-way propagation. In: 76th Ann. Int. Mtg. Soc. Expl. Geophys., Expanded Abstracts, 2333-2337.

Yoon, K., and K. Marfurt (2006), Reverse-time migration using the Poynting vector, Explor. Geophys. 37, 1, 102-107, DOI: 10.1071/EG06102.

Yoon, K., K.J. Marfurt and W. Starr (2004), Challenges in reverse-time migration. In: 74th Ann. Int. Mtg. Soc. Expl. Geophys. Expanded Abstracts, 10571060 .

Yoon, K., M. Guo, J. Cai, and B. Wang (2011), 3D RTM angle gathers from source wave propagation direction and dip of reflector. In: 81st Ann Int. Mtg. Soc. Expl. Geophys., Expanded Abstracts, 1057-1060.

Yoshida, H. (1990), Construction of higher order symplectic integrators, Phys. Lett. A 150, 262-268, DOI: 10.1016/0375-9601(90)90092-3.

Youn, O.K., and H. Zhou (2001), Depth imaging with multiples, Geophysics 66, 1, 246-255, DOI: 10.1190/1.1444901.

Zhang, Y., and G. Zhang (2009), One-step extrapolation method for reverse time migration, Geophysics 74, 4, A29-A33, DOI: 10.1190/1.3123476.

Received 4 November 2015

Received in revised form 22 April 2016

Accepted 5 May 2016 